HEP / 123-qed

\title{
Relativistic predictions of quasielastic proton-nucleus spin observables based on a complete Lorentz invariant representation of the NN scattering matrix
}

\author{
B.I.S. van der Ventel, G.C. Hillhouse, and P.R. De Kock \\ Physics Department, University of Stellenbosch, Stellenbosch, 7600, South Africa
}

(November 2, 2018)

\begin{abstract}
Within the framework of the relativistic plane wave impulse approximation (RPWIA), complete sets of quasielastic $\left(\vec{p}, \vec{p}^{\prime}\right)$ and $(\vec{p}, \vec{n})$ spin observables are calculated employing a general Lorentz invariant representation of the NN scattering matrix (referred to as the IA2 representation). The use of a complete representation eliminates the arbitrariness of a previously-used five-term parameterization (commonly called the IA1 representation) and allows for the correct incorporation of effective-mass-type medium effects within the RPWIA framework and within the context of the Walecka model. For quasielastic scattering from a ${ }^{40} \mathrm{Ca}$ target at incident proton energies between 200 and $500 \mathrm{MeV}$, we investigate the sensitivity of complete sets of spin observables to effective nucleon masses for both IA1 and IA2 representations. In general it is seen that the IA1 representation may overestimate the importance of nuclear medium effects, whereas the IA2-based predictions nearly correspond to values for free nucleon-nucleon scattering.

24.10.Jv, 24.70.+s, 25.40.-h
\end{abstract}

Typeset using REVTEX 


\section{INTRODUCTION}

In a previous paper we developed a relativistic plane wave model for studying medium modifications of the nucleon-nucleon (NN) interaction via complete sets of spin observables for quasielastic $\left(\vec{p}, \vec{p}^{\prime}\right)$ and $(\vec{p}, \vec{n})$ scattering $[1]$. A systematic survey of the predictive power of the latter model compared to experimental data will be presented in this paper.

The main aspect of our model is the use of a general Lorentz invariant representation of the NN scattering matrix referred to as the IA2 representation. This complete expansion of the interaction matrix contains 44 independent invariant amplitudes consistent with parity and time-reversal invariance as well as charge symmetry together with the on-mass-shell condition for the external nucleons [2,3]. Five of the 44 amplitudes are determined from free NN scattering data and are therefore identical to the amplitudes employed in the previously-used five-term parameterization of the NN scattering matrix referred to as the IA1 representation. The remaining 39 amplitudes may be obtained via solution of the Bethe-Salpeter equation employing a one-boson exchange model (with pseudovector pion-nucleon coupling) for the NN interaction [2 [1]. The use of a complete set of NN amplitudes eliminates ambiguities inherent in the IA1 representation. The effect of the nuclear medium on the scattering wave functions is incorporated by replacing free nucleon masses in the Dirac spinors with smaller effective projectile and target nucleon masses within the context of the relativistic mean field approximation of Serot and Walecka [6]. Experimental data on quasielastic spin observables suggest that nuclear shell effects are unimportant, and hence the target nucleus is treated as a non-interacting Fermi gas.

One of the great triumphs of Dirac phenomenology has been the successful prediction

of the analyzing power for quasielastic ${ }^{40} \mathrm{Ca}\left(\vec{p}, \vec{p}^{\prime}\right)$ scattering at $500 \mathrm{MeV}$ based on the IA1 representation of the $\mathrm{NN}$ interaction within the framework of a simple relativistic plane wave model [7]. The latter success is achieved by replacing free nucleon masses with effective nucleon masses in the Dirac spinors, thus enhancing the lower components of the Dirac spinors and resulting in a reduction of the analyzing power relative to the value for 
free scattering: this reduction has been called a "relativistic signature" since no mechanism has been found for its explanation within the framework of the conventional nonrelativistic Schrödinger equation. Despite the successful prediction of the analyzing power, however, the relativistic IA1-based model yields inconsistencies in the sense that quasielastic $\left(\vec{p}, \vec{p}^{\prime}\right)$ and $(\vec{p}, \vec{n})$ spin observables prefer different five-term representations of the NN scattering matrix. As already explained, a more rigorous and unambiguous approach must be based on the IA2 representation of the scattering matrix within the relativistic plane wave impulse approximation. In Ref. [1] we showed that the inclusion of effective masses within the IA2 representation fails to reproduce the large quenching effect predicted by the IA1 representation for the ${ }^{40} \mathrm{Ca}\left(\vec{p}, \vec{p}^{\prime}\right)$ analyzing power at $500 \mathrm{MeV}$. Hence, we concluded that any large deviations of spin observables relative to the corresponding free values are merely artifacts of using an incorrect IA1 representation of the NN scattering matrix, and consequently other effects, such as distortions and multiple scattering, should be considered as possible candidates for reproducing the $500 \mathrm{MeV}$ analyzing power within the IA2 representation.

The question now arises as to how IA2-based predictions compare to data at energies lower than $500 \mathrm{MeV}$ for a range of scattering angles, and how do they compare to the corresponding IA1-based predictions. In principle all calculations should be based on the more rigorous IA2 representation, however, for comparison to previous predictions, the IA1-based calculations are included. In addressing the above questions, we attempt to fully understand the role of effective-mass-type medium effects on spin observables before attempting to incorporate additional effects into our relativistic model. The aim of this paper, therefore, is to perform a systematic study of the predictive power of IA2-based model compared to the published quasielastic polarization data listed in Table \&. The following questions will also be addressed:

- How successful is the effective mass concept, inherent to Dirac phenomenology, in describing quasielastic $\left(\vec{p}, \vec{p}^{\prime}\right)$ and $(\vec{p}, \vec{n})$ scattering data?

- How do numerical results based on the IA2 representation of the NN scattering matrix 
compare to those utilizing the incomplete (and therefore ambiguous) IA1 representation?

In Sec. II the sensitivity of complete sets of quasielastic $\left(\vec{p}, \vec{p}^{\prime}\right)$ and $(\vec{p}, \vec{n})$ spin observables is investigated with respect to a range of different effective projectile and target nucleon masses for both IA1- and IA2-based models. In addition, calculations based on optimal combinations of effective projectile and target nucleon masses are also compared to spin observable data at the centroid of the quasielastic peak. Our main conclusions are presented in Sec. [II].

\section{SENSITIVITY OF SPIN OBSERVABLES TO EFFECTIVE MASSES}

In Ref. [1] it was shown that an IA2-based prediction fails to reproduce the ${ }^{40} \mathrm{Ca}\left(\vec{p}, \vec{p}^{\prime}\right)$ analyzing power at an incident energy of $500 \mathrm{MeV}$. In order to give an initial feeling for the predictive power of our model, the latter reference employed values of the effective nucleon masses which were theoretically extracted by Hillhouse and De Kock [8]. However, the question arises as to whether other combinations of physically acceptable effective projectile and target nucleon masses exist, which provide a better description of the analyzing power. Furthermore, one can also ask whether the latter combination still provides a good description of all the other spin observables, and if not, whether one can find a combination of physically acceptable effective masses which reproduce a complete set of spin observables.

Table $[$ lists all the reactions for which calculations are done. In this paper we only

present the results for the ${ }^{40} \mathrm{Ca}$ target since this is representative of the results which were obtained for all the other target nuclei. Results for the last four reactions can be found in Ref. [15]. Complete sets of spin observable data exist for all the energies and targets used, except ${ }^{40} \mathrm{Ca}(\vec{p}, \vec{n})$ at $T_{l a b}=495 \mathrm{MeV}$ for which no analyzing power data are available and ${ }^{40} \mathrm{Ca}\left(\vec{p}, \vec{p}^{\prime}\right)$ at $T_{l a b}=200 \mathrm{MeV}$ for which only $A_{y}$ and $D_{n n}$ data are available. The reaction ${ }^{40} \mathrm{Ca}(\vec{p}, \vec{n})$ at $T_{l a b}=495 \mathrm{MeV}$ is included since data exist at two different laboratory scattering angles and furthermore it is complementary to the reaction ${ }^{40} \mathrm{Ca}\left(\vec{p}, \vec{p}^{\prime}\right)$ 
at $T_{l a b}=500 \mathrm{MeV}$. The $\left(\vec{p}, \vec{p}^{\prime}\right)$ data at $T_{l a b}=200 \mathrm{MeV}$ are complementary to the $(\vec{p}, \vec{n})$ data at $T_{l a b}=200 \mathrm{MeV}$ and are therefore also included.

\section{A. Effective mass bands}

To answer the above questions, we introduce the concept of an effective mass band in this section, which serves to demonstrate the sensitivity of spin observables to different combinations of effective masses for projectile and target nucleons for both IA1- and IA2-based models. In principle the effective masses can be calculated theoretically following a procedure similar to that outlined in Ref. [8], however, the effective masses are now considered as free parameters which are varied, in step sizes of 0.01 , over the following range of physically acceptable values:

$$
(0.50 ; 0.50) \leq\left(\frac{M_{1}}{M} ; \frac{M_{2}}{M}\right) \leq(1.0 ; 1.0) .
$$

$M$ denotes the free nucleon mass, and $M_{1}$ and $M_{2}$ the effective projectile and target nucleon masses respectively. The lower limit of 0.50 corresponds to the effective nucleon mass in infinite nuclear matter [6]. For the purpose of this exercise we focus on values of the spin observables at an excitation energy corresponding to the centroid of the quasielastic peak in the unpolarized inclusive excitation spectrum. For different laboratory scattering angles empirical data for quasielastic spin observables are relatively constant as a function of nuclear excitation energy at the momentum transfers of interest $\left(|\vec{q}|>0.5 \mathrm{fm}^{-1}\right)$. Hence the trends displayed by observables at the quasielastic peak will be representative of the behavior of spin observables as a function of the energy transferred to the nucleus.

We now introduce the concept of an effective mass band for a particular reaction at a

fixed incident energy as a function of laboratory scattering angle. Let $D_{i^{\prime} j}\left(\omega, \theta_{l a b}, \frac{M_{1}}{M}, \frac{M_{2}}{M}\right)$ denote a particular spin observable from the complete set $\left\{A_{y}, D_{\ell^{\prime} \ell}, D_{s^{\prime} s}, D_{\ell^{\prime} s}, D_{s^{\prime} \ell}, D_{n n}\right\}$ with $D_{0 n} \equiv A_{y}$, where $\omega$ is the energy transferred to the nucleus and $\theta_{l a b}$ is the laboratory scattering angle. For the IA2-based model, the procedure for calculating quasielastic spin 
observables is outlined in Ref. [1]. For the IA1 representation of the NN scattering matrix we employ the phenomenological Horowitz-Love-Franey [16] model with pseudovector pionnucleon coupling as explained in Ref. [8]. In order to do the IA1 calculations, new HorowitzLove-Franey parameters were generated for the energy range of 80 to $195 \mathrm{MeV}$ in steps of $5 \mathrm{MeV}$ [19], and for laboratory energies higher than $200 \mathrm{MeV}$ we employed the Maxwell parameterization of the NN amplitudes 17, 18].

In order to generate the effective mass bands, the spin observables are first calculated as a function of $\omega$ (for fixed $\theta_{l a b}$ ), and then the value of the particular spin observable is extracted at the quasielastic peak, i.e.

$$
D_{i^{\prime} j}^{(p e a k)}\left(\theta_{l a b}, \frac{M_{1}}{M}, \frac{M_{2}}{M}\right)=D_{i^{\prime} j}\left(\omega=\omega_{\text {peak }}, \theta_{l a b}, \frac{M_{1}}{M}, \frac{M_{2}}{M}\right) .
$$

where $\omega_{\text {peak }}$ is the experimental value of the energy transfer associated with the centroid of the quasielastic peak. For a fixed $\theta_{l a b}$, each spin observable is calculated successively for each of the different effective mass combinations in Eq. (2.1). This is repeated for $10^{\circ} \leq \theta_{l a b} \leq 60^{\circ}$ and therefore each effective mass combination generates a curve as a function of $\theta_{l a b}$. Instead of plotting all the different curves on one graph, we calculate, for a fixed $\theta_{l a b}$, the minimum and maximum values for a particular spin observable:

$$
\begin{aligned}
& \left(D_{i^{\prime} j}^{(\text {peak })}\right)_{\text {min }}\left(\theta_{l a b}\right)=\operatorname{Min}\left[D_{i^{\prime} j}^{(\text {peak })}\left(\theta_{l a b}, 1.0 ; 0.5\right) ; D_{i^{\prime} j}^{(\text {peak })}\left(\theta_{l a b}, 1.0 ; 0.6\right) ; \cdots D_{i^{\prime} j}^{(\text {peak })}\left(\theta_{l a b}, 1.0 ; 1.0\right)\right] \\
& \left(D_{i^{\prime} j}^{(\text {peak })}\right)_{\text {max }}\left(\theta_{l a b}\right)=\operatorname{Max}\left[D_{i^{\prime} j}^{(\text {peak })}\left(\theta_{l a b}, 1.0 ; 0.5\right) ; D_{i^{\prime} j}^{(\text {peak })}\left(\theta_{l a b}, 1.0 ; 0.6\right) ; \cdots D_{i^{\prime} j}^{(\text {peak })}\left(\theta_{l a b}, 1.0 ; 1.0\right)\right] .
\end{aligned}
$$

As $\theta_{l a b}$ varies between $10^{\circ}$ and $60^{\circ}\left(D_{i^{\prime} j}^{(\text {peak })}\right)_{\text {min }}\left(\theta_{l a b}\right)$ traces out a lower curve and $\left(D_{i^{\prime} j}^{(\text {peak })}\right)_{\text {max }}\left(\theta_{\text {lab }}\right)$ traces out an upper curve on the graph. All effective mass combinations given by Eq. (2.1) lie between these limits, and this (as a function of scattering angle) forms an effective mass band for each spin observable. Effective mass bands for both IA1 and IA2 representations of the relativistic NN scattering matrix are presented in Figs. 11 to 廿 1 for $\left(\vec{p}, \vec{p}^{\prime}\right)$ and $\left(\vec{p}, \vec{p}^{\prime}\right)$ scattering from a ${ }^{40} \mathrm{Ca}$ nucleus at incident energies of 200 and 500 MeV. Similar figures for the other reactions listed in Table \ can be found in Ref. [15]. The energy range is chosen to correspond to polarized proton energies of interest to experimental programs at facilities such as the National Accelerator Centre (Faure, South Africa) and 
The Research Center for Nuclear Physics (Osaka, Japan). The IA1 and IA2-based effective mass bands are denoted by the straight-line-hatch and dotted-hatch patterns respectively. The solid circles represent the experimental values extracted at the quasielastic peak for a specific laboratory scattering angle: the data are taken from references cited in Table $\mathbb{q}$.

The effective mass bands for the different reactions in Figs. [1 to [ 7 are self-explanatory: if a data point falls outside a band, it means that no effective mass combination can describe that particular point; Rather one must consider other effects such as distortions, multiple scattering or recoil effects in an attempt to reproduce the data. The width of a band also gives an indication of the expected medium effect on a particular spin observable; If the band is wide, then this spin observable is sensitive to a variation in effective masses and it may exhibit a large deviation from the free mass calculation, i.e. a large medium effect. Vice versa if the band is very narrow. The advantage of the effective mass band plots is that they immediately give an indication of whether a particular spin observable can be described via the concept of an effective-mass.

Although Figs. 1 to 14 speak for themselves, we briefly highlight the main results. For both $\left(\vec{p}, \vec{p}^{\prime}\right)$ and $(\vec{p}, \vec{n})$ scattering the IA1 bands are broader than the IA2 bands, indicating the that the IA1 representation severely overestimates the role of effective-mass-type medium effects for quasielastic scattering. In addition, as the energy is lowered, the IA1 bands become broader for $\left(\vec{p}, \vec{p}^{\prime}\right)$ scattering. For $\left(\vec{p}, \vec{p}^{\prime}\right)$ scattering at $200 \mathrm{MeV}$ (Fig. 2) both representations fail to describe $A_{y}$ and $D_{n^{\prime} n}$ indicating that other effects (other than effective-mass-type effects) may play a more important role at low incident energies. Note that for $\left(\vec{p}, \vec{p}^{\prime}\right)$ scattering at both 200 and $500 \mathrm{MeV}$ (Figs. [1 and 2) the IA2-based model fails to reproduce the $A_{y}$ and $D_{n n}$ data. Fig. $⿴$ for $(\vec{p}, \vec{n})$ scattering at $200 \mathrm{MeV}$ clearly illustrates the danger of interpreting medium effects within the IA1 representation: the band for the ambiguous IA1 representation includes the data points for both $D_{s^{\prime} l}$ and $D_{l^{\prime} s}$ spin observables, whereas the more rigorous IA2-based band excludes these data points. 


\section{B. Optimal effective mass combinations}

Next we extract that combination of effective projectile and target nucleon masses which best describes a complete set of spin observables for a range of scattering angles at a fixed incident energy. The systematics of these so-called optimal effective masses is studied for both IA1- and IA2-based models and also compared to values calculated from empirical scalar potentials in an eikonal approximation [8].

We start by defining:

$$
\Delta\left(\frac{M_{1}}{M}, \frac{M_{2}}{M}\right)=\sum_{i=1}^{n_{1}} \sum_{j=1}^{n_{2}}\left(w_{\text {theory }}^{(j)}\left(\theta_{i}\right)-w_{\text {expr }}^{(j)}\left(\theta_{i}\right)\right)^{2}
$$

where $w_{\text {theory }}^{(j)}\left(\theta_{i}\right)$ is the theoretical value of the spin observable evaluated at the laboratory scattering angle $\theta_{i}$ at which the experimental data are available. Similarly $w_{\text {expr }}^{(j)}\left(\theta_{i}\right)$ is the experimental value of the spin observable. $n_{1}$ and $n_{2}$ denote the number of laboratory scattering angles at which data exist and the number of spin observables which were measured, respectively. For example, for the reaction ${ }^{40} \mathrm{Ca}\left(\vec{p}, \vec{p}^{\prime}\right)$ at $T_{l a b}=500 \mathrm{MeV}, n_{1}=1$ (data measured only at one angle $), n_{2}=6\left(A_{y}, D_{\ell^{\prime}, \ell}, D_{s^{\prime} s}, D_{\ell^{\prime}, s}, D_{s^{\prime}, \ell}\right.$ and $\left.D_{n n}\right)$ and $\theta_{i}=19^{\circ}$. Formulae for the calculation of $w_{\text {theory }}^{(j)}\left(\theta_{i}\right)$ can be found in Ref. [1].

The optimal set for a particular reaction is defined as that combination of effective masses for which $\Delta$ is a minimum, i.e. it is that combination of effective masses which best describes all the spin observable data for a particular reaction at a particular energy. Table II

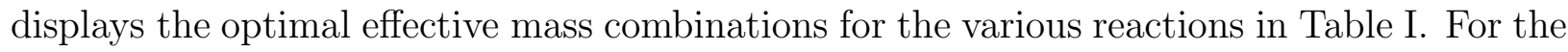

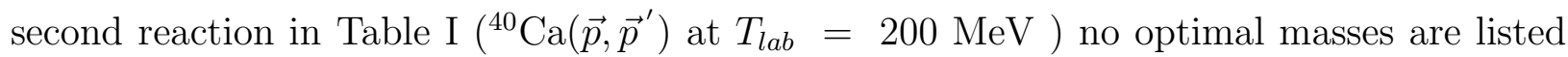
in Table [I] as there were no data on complete sets of observables from which to extract them. For comparison Table [1] also displays the effective mass values calculated in Ref. [8]. Generally one sees that, for both IA1 and IA2-based models, the values of the optimal effective masses agree to within $20 \%$ with the corresponding theoretical values. In addition the optimal effective masses do not exhibit a systematic behavior with respect to target mass and incident energy indicating that one cannot impose a pure plane wave model on 
quasielastic scattering. Additional effects must be included in a more sophisticated model.

In Figs. 1, 3 and 1 we also compare IA1- and IA2-based predictions of spin observables based on the optimal effective masses listed in Table II. The solid and dashed lines denote the IA2 and IA1 predictions respectively. Deviations of the spin observables from the free mass values (long-dash-short-dash) serve as an indication of the importance of effectivemass-type nuclear medium effects for quasielastic scattering. Generally one sees that both optimal IA1 and IA2 predictions are very close to the free mass calculations indicating the insensitivity of quasielastic spin observables to effective-mass-type medium effects.

It is convenient to consider the spin observables in three different groups. Firstly, the spin observables $D_{\ell^{\prime} \ell}, D_{s^{\prime} s}, D_{s^{\prime} \ell}$ and $D_{\ell^{\prime} s}$. For the whole energy range between 200 and 500 $\mathrm{MeV}$ both IA1 and IA2 optimal effective masses provide an adequate description at the quasielastic peak. For the $(\vec{p}, \vec{n})$ observables the description is not as good as for the $\left(\vec{p}, \vec{p}^{\prime}\right)$ observables.

Next we focus on $D_{n n}$. The description of $D_{n n}$ becomes problematic for both $\left(\vec{p}, \vec{p}^{\prime}\right)$ and $(\vec{p}, \vec{n})$ scattering as the energy is lowered. For the $\left(\vec{p}, \vec{p}^{\prime}\right)$ reaction the data point shifts away from the effective mass band as the energy is lowered, while for the $(\vec{p}, \vec{n})$ reaction the theoretical calculation exhibits an oscillatory motion at $495 \mathrm{MeV}$ which causes it to miss the data. At $200 \mathrm{MeV}$ there is still a variation with respect to laboratory scattering angle in the theoretical calculation whereas the data are quite flat. A possible explanation for the latter discrepancy is the exclusion of distortions and recoil effects in our model.

Lastly, the analyzing power $A_{y}$ is considered. In the IA2 representation of the NN scattering matrix the optimal effective mass set does not provide a good description of the $A_{y}$ data at the quasielastic peak for the reaction $\left(\vec{p}, \vec{p}^{\prime}\right)$ at $500 \mathrm{MeV}$. (It may even be better described by some other specially chosen, but realistic pair of effective masses.) Furthermore, as the energy is lowered, the $A_{y}$ data point shifts away from the effective mass band. The $(\vec{p}, \vec{n})$ data for $A_{y}$ are, however, much better described by the optimal IA2 set.

The failure of the IA2-based model to predict $A_{y}$ and $D_{n n}$ for $\left(\vec{p}, \vec{p}^{\prime}\right)$ scattering at 200 $\mathrm{MeV}$ calls for a more sophisticated treatment of nuclear distortions and recoil effects. To this 
end we have developed a relativistic distorted wave model for quasielastic scattering [19]; Numerical results will be presented in a future paper. Furthermore, since that distortions play a more prominent role at low energies, the measurement of a complete set of $\left(\vec{p}, \vec{p}^{\prime}\right)$ spin observables at $200 \mathrm{MeV}$ will be extremely useful for checking the validity of our distorted model. The latter measurements will also complement the existing $(\vec{p}, \vec{n})$ data measured at the Indiana University Cyclotron Facility [12].

Calculations have been performed for all the reactions listed in Table $\mathbb{1}$ as a function of energy transferred to the nucleus: these results are available from the authors on request. Conclusions based on the latter are consistent with the present investigation at the centroid of the quasielastic peak.

\section{CONCLUSION}

In this investigation effective projectile and target nucleon masses were treated as free parameters and it was found that no effective mass combination could describe both $\left(\vec{p}, \vec{p}^{\prime}\right)$ and $(\vec{p}, \vec{n})$ scattering observables. Even though the IA2 treatment of medium effects (within the RPWIA framework) is the most advanced to date, it still fails to describe all observables; the glaring example being the prediction of $A_{y}$ for $\left(\vec{p}, \vec{p}^{\prime}\right)$ scattering as the energy is lowered from $500 \mathrm{MeV}$ to $200 \mathrm{MeV}$. In general it is seen that IA2-based effective-mass predictions are

close to the corresponding free values, whereas the ambiguous IA1 representation severely overestimates the importance of effective-mass-type medium effects. Despite the successes of the Walecka model effective mass concept within the relativistic plane wave impulse approximation, the theoretical work should now start to include additional effects like multiple scattering, recoil effects and distortions of the projectile. A relativistic distorted wave model (initially employing the IA1 representation of the NN scattering matrix) has been presented in Ref. [19, but still needs to be implemented numerically. 


\section{ACKNOWLEDGMENTS}

The authors wish to thank Professor S.J. Wallace (University of Maryland, USA) for providing the IA2 invariant amplitudes used in the present calculations. The financial as-

sistance to B.I.S.v.d.V by the Harry Crossley Foundation, the South African FRD and the National Accelerator Centre is gratefully acknowledged. 


\section{REFERENCES}

[1] B.I.S. van der Ventel, G.C. Hillhouse, P.R. De Kock, and S.J. Wallace, Phys. Rev. C 60, $64618(1999)$.

[2] J.A. Tjon and S.J. Wallace, Phys. Rev. C 32, 1667 (1985).

[3] J.A. Tjon and S.J. Wallace, Phys. Rev. C 35, 1085 (1987).

[4] E.E. van Faassen and J.A. Tjon, Phys. Rev. C 28, 2354 (1983).

[5] E.E. van Faassen and J.A. Tjon, Phys. Rev. C 30, 285 (1984).

[6] B. D. Serot and J. D. Walecka, in Advances in Nuclear Physics, edited by J. W. Negele and E. Vogt (Plenum Press, New York, 1986), Vol. 16, p. 116.

[7] C. J. Horowitz and D. P. Murdock, Phys. Rev. C 37, 2032 (1988).

[8] G.C. Hillhouse and P.R. de Kock, Phys. Rev. C 49, 391 (1994).

[9] T.A. Carey, K.W. Jones, J.B. McClelland, M. Moss, L.B. Rees, N. Takana, and A.D. Bacher, Phys. Rev. Lett 53, 144 (1984).

[10] D.S. Carman, Inclusive and Exclusive Quasiefree $(\vec{p}, N p)$ Reaction Studies from ${ }^{2} H$ and ${ }^{12} \mathrm{C}$ at $200 \mathrm{MeV}$, Ph.D Thesis, Indiana University (1995), unpublished.

[11] T.N. Taddeucci, private communication.

[12] C.L. Hautala, Measurement of Polarization Observables in the Quasielastic Region on ${ }^{n a t} \mathrm{Ca}$ and ${ }^{n a t} \mathrm{~Pb}$ using the $(\vec{p}, \vec{n})$ Reaction at $200 \mathrm{MeV}, \mathrm{Ph} . \mathrm{D}$ Thesis, Ohio University (1998), unpublished and private communication.

[13] C. Chan, T.E. Drake, R. Abegg, D. Frekers, O. Häusser, K. Hicks, D.A. Hutcheon, L. Lee, C.A. Miller, R. Schubank, and S. Yen, Nucl. Phys. A510, 713 (1990).

[14] O. Häusser, R. Abegg, R.G. Jeppesen, R. Sawafta, A. Celler, A. Green, R.L. Helmer, R. Henderson, K. Hicks, K.P.Jackson, J. Mildenberger, C.A. Miller, M.C. Vetterli, S. 
Yen, M.J. Iqbal, and R.D. Smith, Phys. Rev. Lett. 61, 8221988.

[15] B.I.S. van der Ventel, General Lorentz Invariant Representation of the NN Interaction Applied to Quasielastic Scattering of Polarized Protons, Ph.D Thesis, University of Stellenbosch (1999), unpublished.

[16] C.J. Horowitz, Phys. Rev. C 31, 1340 (1985).

[17] O.V. Maxwell, Nucl. Phys. A600, 509 (1996).

[18] O.V. Maxwell, Nucl. Phys. A638, 747 (1998).

[19] G.C. Hillhouse, Relativistic Descriptions of Polarization Transfer Observables for Quasielastic Proton Scattering, Ph.D Thesis, University of Stellenbosch (1999), unpublished. 


\section{FIGURES}

FIG. 1. Values of $A_{y}$ and $D_{i^{\prime} j}$ versus $\theta_{l a b}$ for ${ }^{40} \mathrm{Ca}\left(\vec{p}, \vec{p}^{\prime}\right)$ at $T_{l a b}=500 \mathrm{MeV}$. Solid and dashed lines represent the calculations with optimal effective mass values in respectively the IA2 and IA1

representations. The hatched bands denote the range of values which result from varying $\frac{M_{1}}{M}$ and $\frac{M_{2}}{M}$ over the full range (see text): The straight line hatch pattern denotes the IA1 model; the dotted hatch pattern the IA2 model. The long-dash-short-dash lines represent the free mass values. Data $\left(\right.$ at $\left.\theta_{l a b}=19^{\circ}\right)$ are from Ref. [9].

FIG. 2. For this reaction, ${ }^{40} \mathrm{Ca}\left(\vec{p}, \vec{p}^{\prime}\right)$ at $T_{l a b}=200 \mathrm{MeV}$ and $\theta_{l a b}=30^{\circ}$ only a free mass calculation (denoted by the solid line) was performed due the lack of a complete set of spin observables. The data are form Ref. [10].

FIG. 3. Same as Fig. 1 but for the reaction ${ }^{40} \mathrm{Ca}(\vec{p}, \vec{n})$ at $T_{l a b}=495 \mathrm{MeV}$ and $\theta_{l a b}=18^{\circ}$ and $27^{\circ}$. The data are from Ref. [11].

FIG. 4. Same as Fig. 1 but for the reaction ${ }^{40} \mathrm{Ca}(\vec{p}, \vec{n})$ at $T_{l a b}=200 \mathrm{MeV}$ and $\theta_{l a b}=24^{\circ}$, $37^{\circ}$ and $48^{\circ}$. The data are from Ref. [12]. 


\section{TABLES}

TABLE I. Experimental data for which calculations were done at the quasielastic peak (as a function of laboratory scattering angle) and as a function of energy transfer

\begin{tabular}{|c|c|c|c|}
\hline \hline Reaction & $T_{l a b}(\mathrm{MeV})$ & $\theta_{\text {lab }}$ (degrees) & Reference \\
\hline${ }^{40} \mathrm{Ca}\left(\vec{p}, \vec{p}^{\prime}\right)$ & 500 & 19 & {$[9]$} \\
\hline${ }^{40} \mathrm{Ca}\left(\vec{p}, \vec{p}^{\prime}\right)$ & 200 & 30 & {$[1]$} \\
\hline${ }^{40} \mathrm{Ca}(\vec{p}, \vec{n})$ & 495 & 18,27 & {$[1]$} \\
\hline${ }^{40} \mathrm{Ca}(\vec{p}, \vec{n})$ & 200 & $24,37,48$ & {$[13]$} \\
\hline${ }^{12} \mathrm{C}\left(\vec{p}, \vec{p}^{\prime}\right)$ & 420 & 24 & {$[13]$} \\
\hline${ }^{12} \mathrm{C}\left(\vec{p}, \vec{p}^{\prime}\right)$ & 290 & 30 & {$[1]$} \\
\hline${ }^{54} \mathrm{Fe}\left(\vec{p}, \vec{p}^{\prime}\right)$ & 290 & 20 & {$[12]$} \\
\hline${ }^{208} \mathrm{~Pb}(\vec{p}, \vec{n})$ & 200 & $24,37,48$ & {$[13]$} \\
\hline \hline
\end{tabular}


TABLE II. Values of optimal effective mass combinations, $\left(\frac{M_{1}}{M}, \frac{M_{2}}{M}\right)$, extracted at the quasielastic peak. The last column refers to the effective mass combinations which are calculated theoretically [18].

\begin{tabular}{|l|l|ll|ll|ll|}
\hline \hline Reaction & $T_{l a b}(\mathrm{MeV})$ & \multicolumn{2}{|c|}{ IA1 } & \multicolumn{2}{|c|}{ IA2 } & \multicolumn{2}{c|}{ Theory } \\
\hline & & $\frac{M_{1}}{M}$ & $\frac{M_{2}}{M}$ & $\frac{M_{1}}{M}$ & $\frac{M_{2}}{M}$ & $\frac{M_{1}}{M}$ & $\frac{M_{2}}{M}$ \\
\hline${ }^{40} \mathrm{Ca}\left(\vec{p}, \vec{p}^{\prime}\right)$ & 500 & 0.96 & 0.96 & 1.0 & 0.86 & 0.89 & 0.82 \\
\hline${ }^{40} \mathrm{Ca}(\vec{p}, \vec{n})$ & 495 & 0.75 & 0.75 & 0.85 & 0.85 & 0.89 & 0.82 \\
\hline${ }^{40} \mathrm{Ca}(\vec{p}, \vec{n})$ & 200 & 1.0 & 0.87 & 0.93 & 0.92 & 0.83 & 0.75 \\
\hline${ }^{12} \mathrm{C}\left(\vec{p}, \vec{p}^{\prime}\right)$ & 420 & 0.88 & 0.88 & 0.88 & 0.76 & 0.86 & 0.79 \\
\hline${ }^{12} \mathrm{C}\left(\vec{p}, \vec{p}^{\prime}\right)$ & 290 & 0.93 & 0.93 & 1.0 & 0.92 & 0.83 & 0.77 \\
\hline${ }^{54} \mathrm{Fe}\left(\vec{p}, \vec{p}^{\prime}\right)$ & 290 & 0.87 & 0.87 & 0.86 & 0.74 & 0.83 & 0.77 \\
\hline${ }^{208} \mathrm{~Pb}(\vec{p}, \vec{n})$ & 200 & 0.94 & 0.84 & 0.94 & 0.94 & 0.85 & 0.83 \\
\hline \hline
\end{tabular}



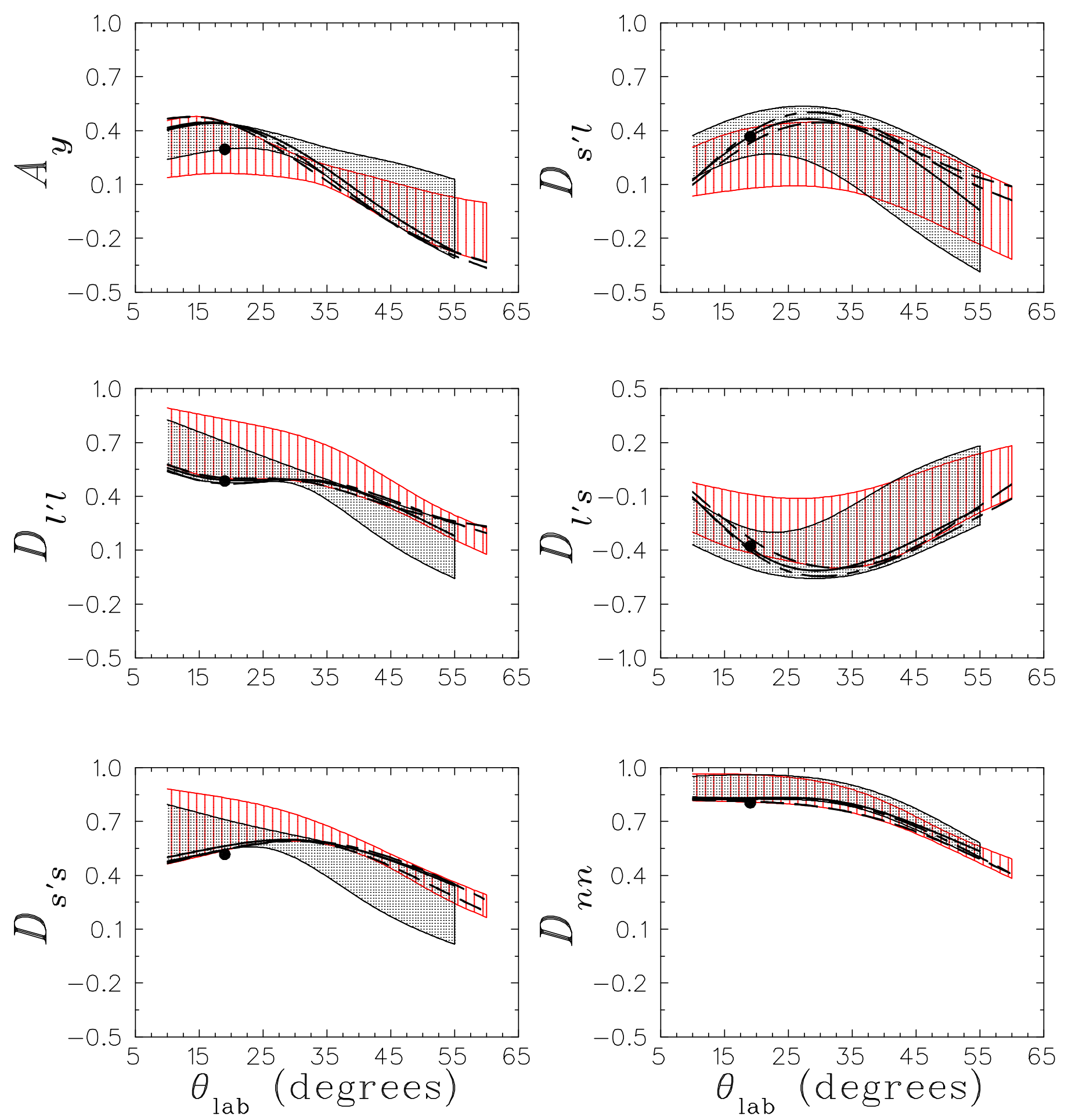

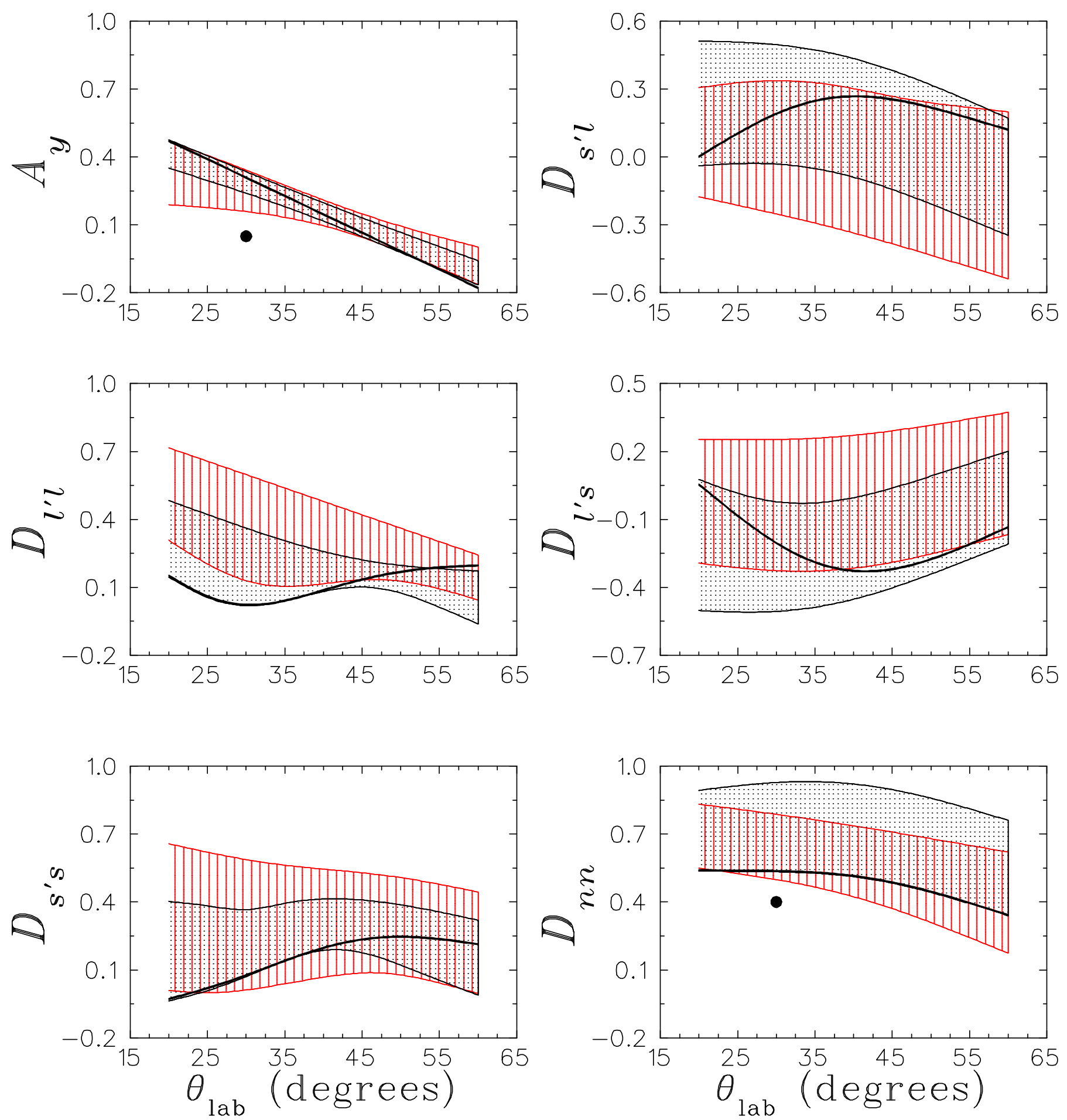

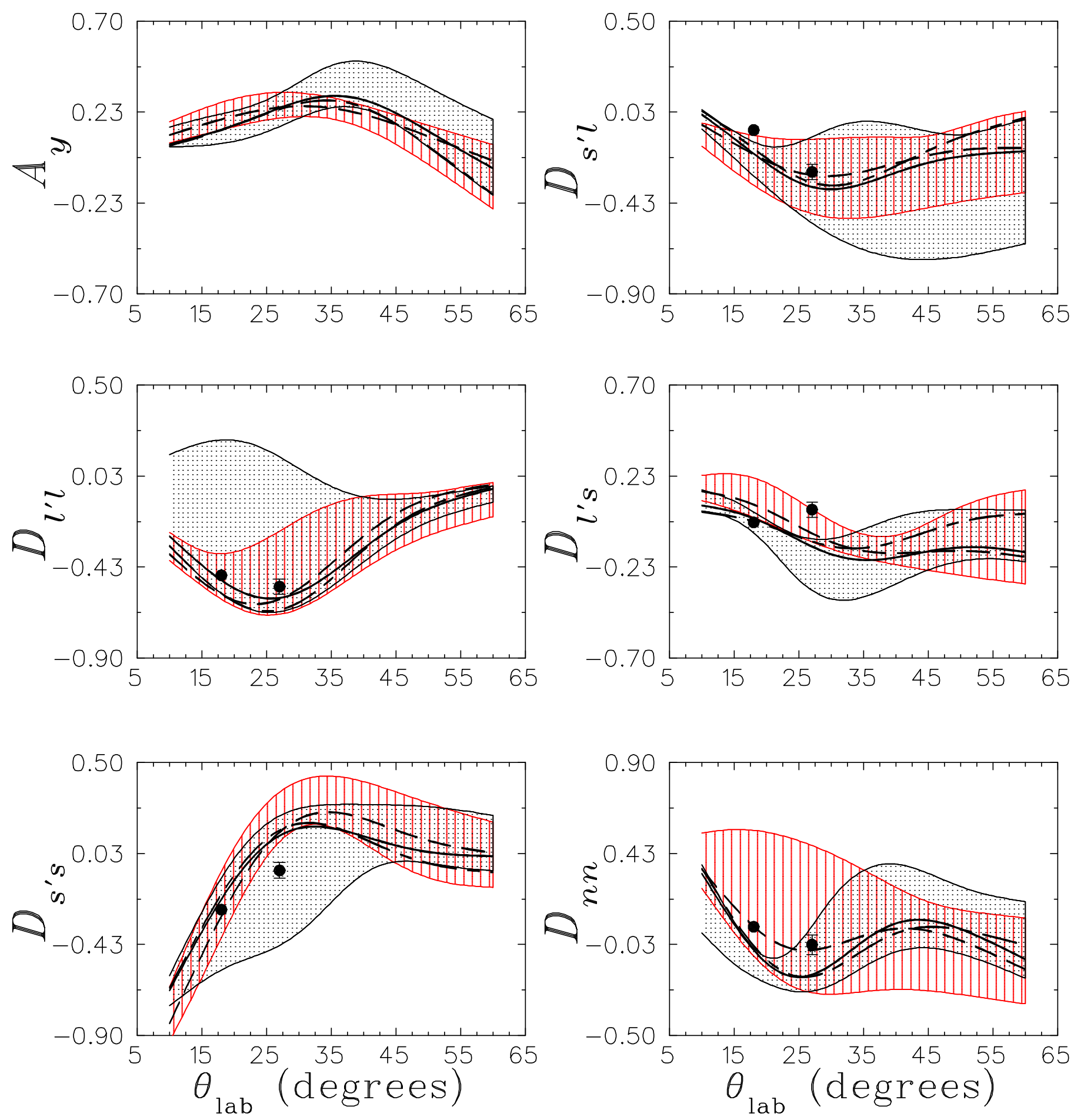

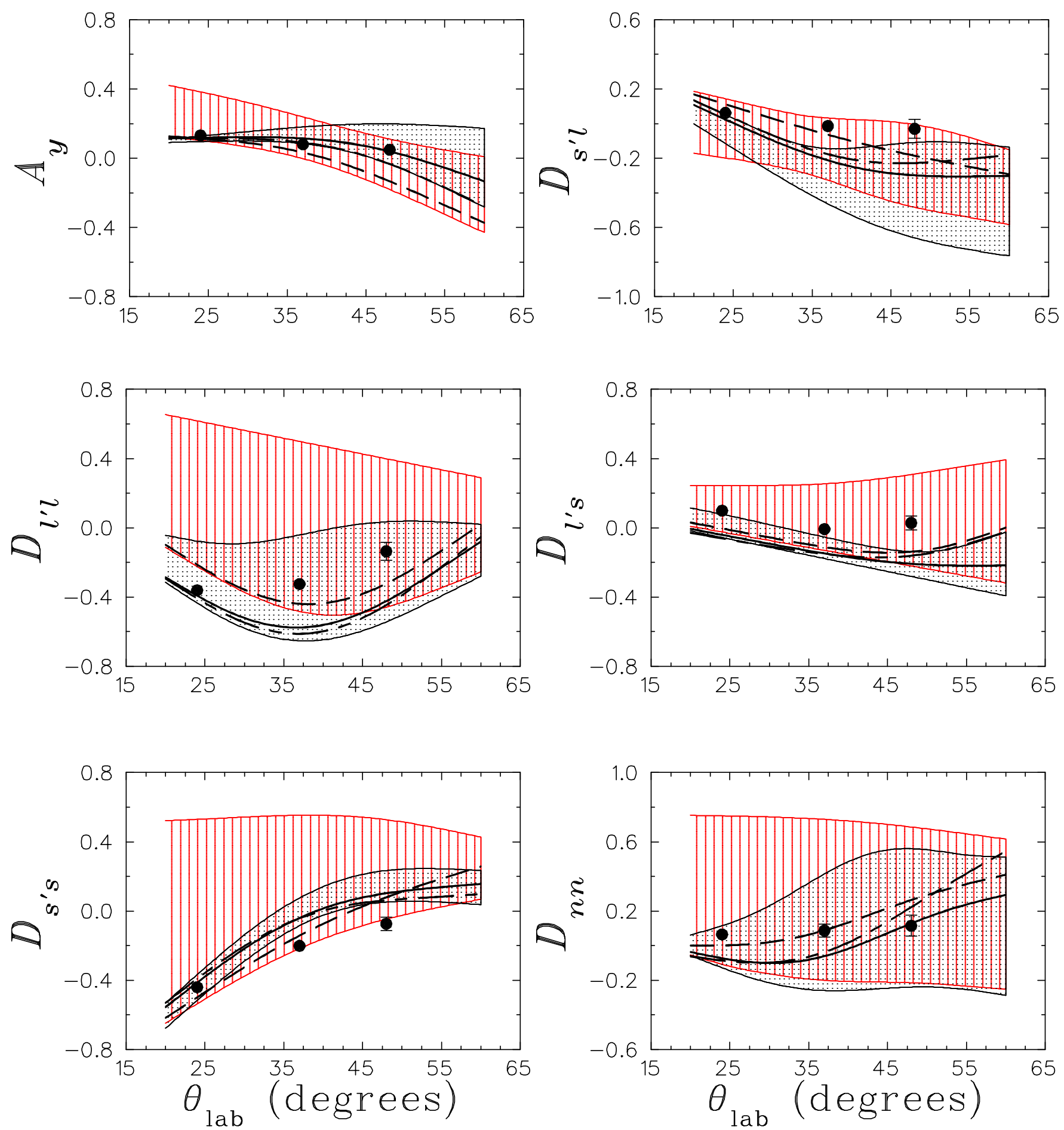